\title{
Inhibitory bacteria reduce fungi on early life stages of endangered Colorado boreal toads (Anaxyrus boreas)
}

\author{
Jordan G Kueneman ${ }^{1}$, Douglas C Woodhams ${ }^{1,3}$, Will Van Treuren ${ }^{2,4}$, Holly M Archer ${ }^{1}$, \\ Rob Knight ${ }^{2,5}$ and Valerie J McKenzie ${ }^{1}$ \\ ${ }^{1}$ Department of Ecology and Evolutionary Biology, University of Colorado, Boulder, CO, USA and \\ ${ }^{2}$ BioFrontiers Institute, University of Colorado, Boulder, CO, USA
}

\begin{abstract}
Increasingly, host-associated microbiota are recognized to mediate pathogen establishment, providing new ecological perspectives on health and disease. Amphibian skin-associated microbiota interact with the fungal pathogen, Batrachochytrium dendrobatidis $(B d)$, but little is known about microbial turnover during host development and associations with host immune function. We surveyed skin microbiota of Colorado's endangered boreal toads (Anaxyrus boreas), sampling 181 toads across four life stages (tadpoles, metamorphs, subadults and adults). Our goals were to (1) understand variation in microbial community structure among individuals and sites, (2) characterize shifts in communities during development and (3) examine the prevalence and abundance of known $B d$-inhibitory bacteria. We used high-throughput 16S and 18S rRNA gene sequencing (Illumina MiSeq) to characterize bacteria and microeukaryotes, respectively. Life stage had the largest effect on the toad skin microbial community, and site and $B d$ presence also contributed. Proteobacteria dominated tadpole microbial communities, but were later replaced by Actinobacteria. Microeukaryotes on tadpoles were dominated by the classes Alveolata and Stramenopiles, while fungal groups replaced these groups after metamorphosis. Using a novel database of $B d$-inhibitory bacteria, we found fewer $B d$-inhibitory bacteria in post-metamorphic stages correlated with increased skin fungi, suggesting that bacteria have a strong role in early developmental stages and reduce skinassociated fungi.
\end{abstract}

The ISME Journal (2016) 10, 934-944; doi:10.1038/ismej.2015.168; published online 13 November 2015

\section{Introduction}

Throughout their life cycle, amphibians are exposed to diverse pathogenic threats including bacteria, viruses, fungi, protozoans and helminths. Amphibians defend themselves with innate and adaptive immune systems, behavioral strategies, and symbiotic cutaneous and internal microbial communities (Rollins-Smith et al., 2011). The function of the community of cutaneous microbiota in the health of larval amphibians is unknown. For some amphibian species, the microbial environment is manipulated before they emerge from the egg. Evidence of

Correspondence: J Kueneman, Department of Ecology and Evolutionary Biology, University of Colorado, Ramaley N-122, UCB 334 Boulder, CO 80309, USA.

E-mail: Jordan.kue@gmail.com

${ }^{3}$ Current address: Department of Biology, University of Massachusetts, Boston, MA, USA.

${ }^{4}$ Current address: Department of Microbiology and Immunology, Stanford University, Palo Alto, CA, USA.

${ }^{5}$ Current address: Department of Biomedical Sciences, University of California, San Diego, CA, USA.

Received 29 March 2015; revised 21 July 2015; accepted 31 July 2015; published online 13 November 2015 transmission of antifungal bacteria from adult to egg has been observed for the glass frog Hyalinobatrachium colymbiphyllum (Walke et al., 2011) and the salamanders Plethodon cinereus and Hemidactylium scutatum (Brucker et al., 2008; Banning et al., 2008). These examples suggest that the assembly of microbial symbionts in early life stages may have adaptive significance for adult host health. The role of symbiotic microbes in defending against pathogens affecting amphibians is an area of intense interest (Harris et al., 2009; Woodhams et al., 2015), although studies to date have primarily focused on post-metamorphic amphibians. The process of symbiont community assembly through host development for both pre- and post-metamorphosis has not been explored and is the aim of the current study.

When and how the symbiotic microbes perform key defensive functions during the different life stages of amphibians is important for understanding the larger role of the microbes in amphibian health. In vertebrate hosts, microbial symbionts play vital roles in educating the innate and acquired immune systems (Iwasaki and Ruslan, 2010; Hooper et al., 2012), and defend the host directly against pathogens prior 
to the development of a fully functional immune system (Rautava et al., 2012). For amphibians with biphasic life history strategies, the immune system is restructured during metamorphosis. Larval amphibians (for example, tadpoles) have innate immune cells in the form of lymphocytes, which decline during metamorphosis and then reestablish postmetamorphosis (Rollins-Smith, 1998). Many immune cells begin forming in organs absent in tadpoles, such as the kidney and bone marrow (Rollins-Smith, 1998). The adaptive immune functions increase significantly after metamorphosis, including MHC class 1 and 2 antigen recognition domains (Rollins-Smith, 1998). Consequently, skin-associated microbial communities and the innate immune system may serve as the primary lines of defense against pathogens for larval amphibians before the adaptive immune system is fully functional. The mechanisms underlying how host species select and maintain bacterial communities on the skin are not yet fully understood (Walke et al., 2014), but host immune systems mediate microbial interactions in many ways (Tennessen et al., 2009). In amphibians, mucus glands produce secretions that act as a physical protective barrier and substrate for resident microbiota (Rollins-Smith et al., 2009; Woodhams et al., 2014). Driven by the innate immune system in early life stages, defensive glands in the skin produce antimicrobial peptides, alkaloids and lysozymes, which limit the growth of some bacterial taxa and promote growth of others (Woodhams et al., 2014). Post metamorphosis, amphibians reorganize the adaptive immune system, which can mount the production of $B$ and $\mathrm{T}$ lymphocytes and antigen-specific antibodies that can distinguish beneficial microbiota and self from nonself (Rollins-Smith, 1998). To determine which, if any role skin microbial communities play in these transitions in the immune system during development, we must sample these communities across developmental stages.

Of particular interest for amphibian conservation is whether skin microbial communities can protect the host through antibiosis of Batrachochytrium dendrobatidis $(B d)$, the causal agent of the disease chytridiomycosis. Chytridiomycosis is a leading cause of amphibian declines worldwide (Kilpatrick et al., 2010; Bletz et al., 2013). We therefore investigated the skin-associated microbial communities of boreal toads (Anaxyrus boreas; previously Bufo boreas), a $B d$ susceptible species. The boreal toad inhabits much of the Western United States and Canada, ranging from Baja California to southeastern Alaska (Muths and Guyer, 2003). Boreal toads are long-lived amphibians, with lifespans up to 12 years (Olson, 1992). Larval aquatic tadpole stages metamorphose within one season, and post-metamorphic subadults can take up to 4 years to become sexually mature adults (Scherff-Norris, 1997). A. boreas has suffered severe population declines in portions of its southeastern range, and has been placed under protective legislation dictated by its conservation status. This status includes 'endangered' in Colorado and New Mexico (Goettl, 1997). The primary driver of $A$. boreas decline in Colorado is chytridiomycosis caused by $B d$ (Muths and Guyer, 2003; Scherer et al., 2005). Intriguingly, populations of $A$. boreas differ in their response to $B d$ infection. Hypotheses for the basis for these differences include host genetic factors, variable pathogenicity of unique $B d$ strains, secondary or tertiary stressors, toad densities, as well as environmental conditions such as minimum daily air temperature, snow depth and access to refugia (Scherer et al., 2008; Murphy et al., 2009; Savage and Zamudio, 2011). Bacterial communities of the amphibian skin might provide a complementary or alternative explanation for the observed differences in susceptibility to $B d$.

We explore the natural variation of populations of A. boreas collected from Colorado during one season to address the following questions. First, what factors best explain natural variation of the boreal toad skin microbiome? Second, how do microbial communities including bacteria and microeukaryotic organisms vary across boreal toad life stages, and what can we infer about the role of microbial communities through host development? Third, what is the distribution and abundance of known $B d$-inhibitory bacteria across life stages of wild boreal toads?

\section{Materials and methods}

Data collection

During July and August 2012, we sampled boreal toads from 17 locations across western Colorado (Supplementary Table 1, Supplementary Figures 1 and 2). Permits and authorization were granted by Colorado Parks and Wildlife and the University of Colorado Institutional Animal Care and Use Committees IACUC. We surveyed high-elevation $(2200-3400 \mathrm{~m})$ wetlands on both the eastern and western slope (including Larimer, Clear Creek, Grand, Chaffee, Gunnison and Mesa counties) for A. boreas populations. The study design captured geographic variation and distinct life history traits through metamorphosis and development (tadpole, metamorph, sub-adult and adult). Amphibians sampled at each site were all collected on the same day, except for sample-sites Panhandle and Hendersen/Urad, which required additional sampling due to low capture rates. Sampling location and sample size of each amphibian and life history stage are given in Supplementary Table 1. Microbial swab samples were collected, DNA extracted, and processed as in Kueneman et al., 2014. (Supplementary Material). Samples were tested for $B d$, following standard protocols (Supplementary Material). 


\section{Analysis methods}

Methods explaining variation in toad skin microbiomes. To address our first question, regarding factors that explain variation in microbial community structure of toads, we evaluated four factors (sex, life stage, site and $B d$ presence or absence at a site) that we thought might affect the structure of toad bacterial communities. First, we performed a single-factor ANOSIM for each of the four factors as a predictor of boreal toad skin bacterial community similarity. Sex was the only factor that was not a significant predictor, and thus was not considered further in these analyses. Next, we performed a three-factor crossed PERMANOVA with host species, $B d$ presence or absence, and sample site as factors. In this analysis, site was nested within $B d$ presence or absence, because the two terms are not independent. This PERMANOVA included bacterial sequences from the skin of 168 individuals, removing 13 individuals from the total data set because they did not meet our criteria of greater than one individual of particular life stage at a given site, and/or the site had unknown $B d$ presence or absence $(N=2$ sites). Sampling locations of boreal toads in Colorado are provided in a map (Supplementary Figure S2).

Developmental transitions in taxa and alpha diversity To examine the effects of developmental life stage on the skin-associated microbial communities, we used a subset of the data that includes toad individuals from different life stages collected from a single site (Trout Creek). All four life stages were only found at the same location and time point for this endangered species at Trout Creek. For bacterial samples, $n=8$ tadpoles, $n=8$ metamorphs, $n=8$ subadults and $n=10$ adults. For microeukaryotes, $n=5$ tadpoles, $n=4$ metamorphs, $n=5$ subadults and $n=5$ adults. We compared bacteria and microeukaryotes $16 \mathrm{~S}$ and 18S sequences from trout creek (Figures 1a and b, 2a and $b$, 3a and b). The Shannon diversity metric (log base 2) was calculated for both data sets in QIIME. Samples were analyzed for microeukayotes and bacteria. Quantitative PCR of bacteria and fungal DNA equivalents were obtained following Emerson et al., 2015. Co-occurrence network analyses preformed on microeukaryotes and bacteria sequences were obtained within samples $(N=19)$.

\section{Co-occurence networks}

Co-occurrence networks examine interactions between members of a community (Faust and Raes, 2012; Berry and Widder, 2014). Co-occurrence networks can reveal ecologically important correlations in data by testing each pair-wise interaction between features (OTUs) in a data set. Those feature-feature pairs ('edges' of the network) that are deemed to be significantly co-occurring using Monte Carlo are included in the resulting network. Possible ecological reasons for associations among community members include mutualism, shared environmental preference, biochemical similarity and so on. Here, we tested for correlation between eukaryotic and bacterial OTUs at Trout Creek using the co-occurrence detection tool SparCC (Friedman and Alm, 2012). This analysis included 19 toad individuals from Trout Creek for which we had successful sequencing yields for both $16 \mathrm{~S}$ and $18 \mathrm{~S}$ : $n=5$ tadpoles, $n=4$ metamorphs, $n=5$ subadults and $n=5$ adults. Briefly, we created a single OTU table that included the most prevalent eukaryotic and bacterial OTUs by removing OTUs from both data sets found in $50 \%$ or less of samples. We used the default SparCC parameters, with 500 bootstraps, and assigned an edge as significant at a $P$-value of 0.002
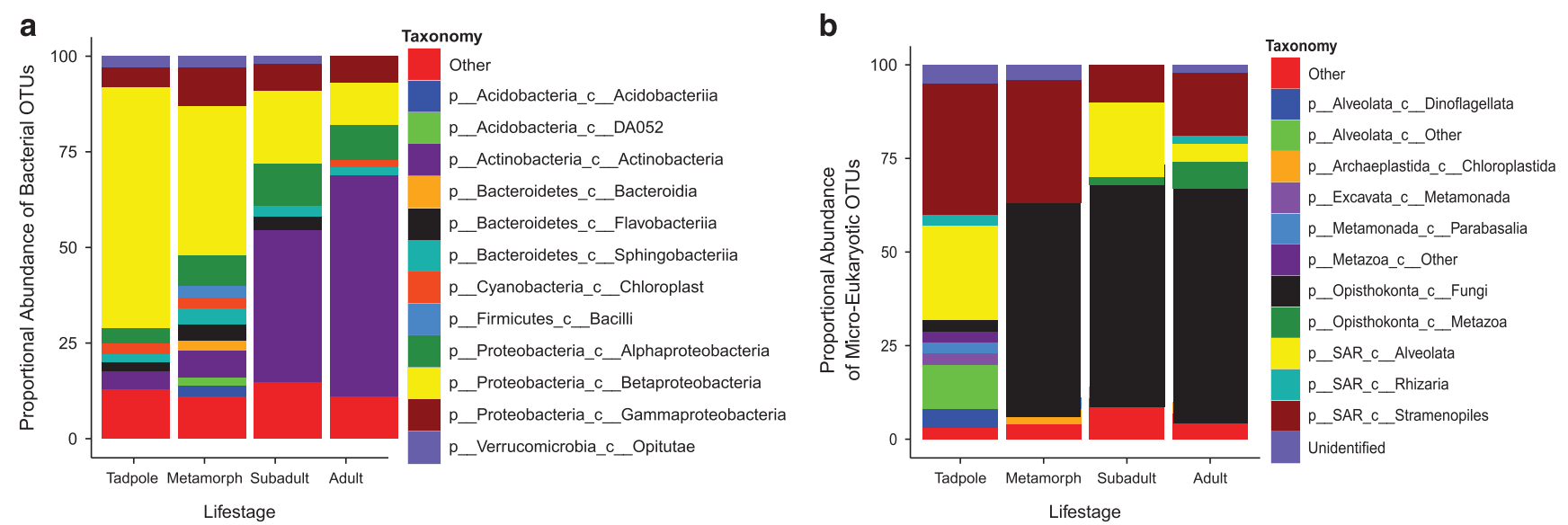

Figure 1 Major skin microbial taxa on boreal toads from Trout Creek at each life stage, collected on the same day. (a) The proportional abundance of bacterial OTU sequences per major taxon across lifestages: tadpoles $(n=8)$, metamorphs $(n=8)$, subadults $(n=8)$ adults $(n=10)$. (b) The proportional abundance of microeukaryote OTU sequences per major taxon across lifestages: tadpoles $(n=5)$, metamorphs $(n=4)$, subadults $(n=5)$, adults $(n=5)$. Data in a is based on $1075016 S$ sequences per sample and data in $\mathbf{b}$ is based on $240018 \mathrm{~S}$ sequences per sample. Taxonomy is designated by $\mathrm{p}=$ phylum and $\mathrm{c}=$ class. 

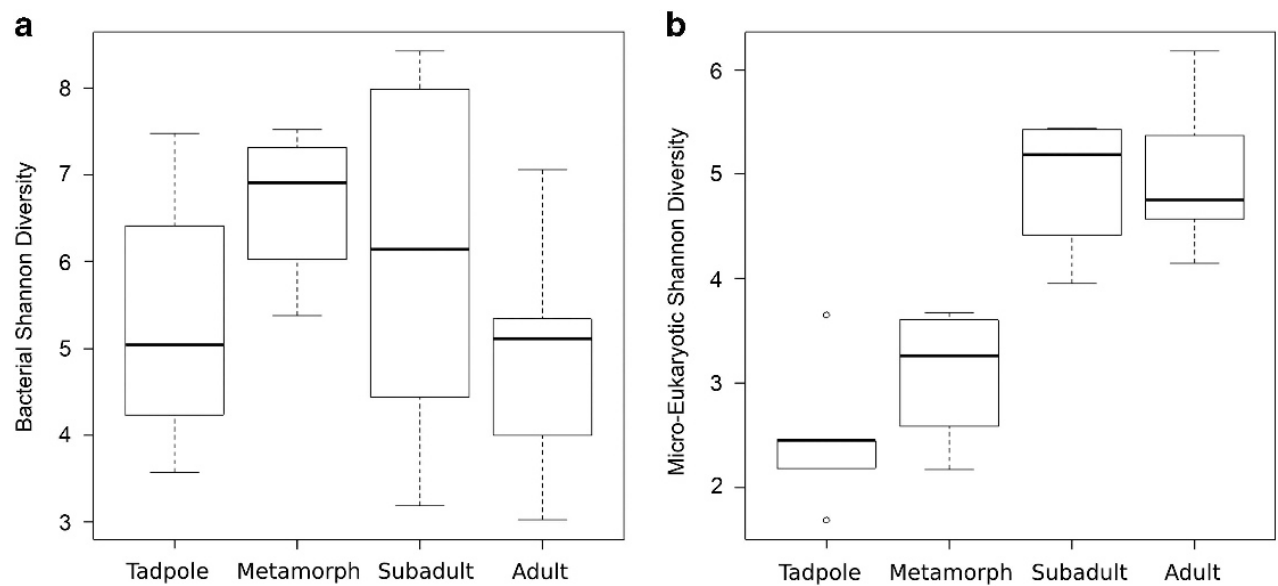

Figure 2 Shannon diversity of microbial communities on boreal toads, by lifestage. (a) Alpha diversity (Shannon) of bacteria by life-stage from Trout Creek $(n=32)$. (b) Alpha diversity (Shannon) of microeukaryotes by life stage from Trout Creek $(n=19)$.

(1 bootstrapped correlation as or more extreme than observed correlation value). Our $P$-value threshold was lower than suggested by SparCC, because our internal testing has suggested that 0.05 is a very permissive threshold that results in large number of false positives. The resulting network had 721 edges, with 210 distinct OTUs (average connectivity $=6.86$, $\max =31, \min =1$ ) 30 of which were eukaryotic. We imported the edge table into Cytoscape 3.0 (Smoot et al., 2011) and performed a springembedded force directed layout with weighting by correlation value. Edges were bundled with default bundling parameters in Cytoscape to improve readability.

Supervised learning and differential abundance testing Supervised learning is a machine learning technique that uses subsets of the samples to build predictive models about sample class membership. We used the Random Forests algorithm supervised learning algorithm to identify taxa that correlated with physiological, developmental and environmental variables. Analysis was conducted using the QIIME script supervised_learning.py with 1000 trees per forest, and 5- or 10-fold cross validation as appropriate for the given sample class sizes. Because random forests select features from the input set stochastically, it will perform poorly if many low abundance and uninformative features are present. Thus, prior to supervised learning, features (OTUs and taxonomically summarized OTUs) found in $10 \%$ of samples or less were removed. Differential abundance testing was performed with the QIIME script group_significance.py. In brief, features (OTUs) were compared for significantly different abundance between samples grouped in a variety of ways (for example, by sex, life stage, location and so on) with a Kruskal-Wallis test. $P$-values were corrected by the FDR method of Benjamini and Hochberg (1995). $P$-values $<0.05$ after correction were classified as significantly different. Prior to differential abundance testing, features were also removed as described for supervised learning.

Bd-inhibitory Bacteria 'OTU' Database and Bd scores We utilized a concurrently developed database of cultured $B d$-inhibitory bacteria (referred to here as the 'inhibitory database'), containing $125516 \mathrm{~S}$ rRNA gene sequences isolated from amphibian skin (Woodhams et al., 2015). Many studies have tested these isolates for bioactivity against fungal pathogens including Bd, Mariannaea elegans and Rhizomucor variabilis in coculture challenge assays, summarized by Woodhams et al (2015). From this database, we generated OTU lists by clustering sequences at $97 \%$ similarity using the Greengenes August 2013 reference. The sequence matching algorithm used to find matches from our toad data set with the 'inhibitory database' could potentially be influenced by which method is used, ranging from strict matches to predicted phylogenetically similar matches. For this reason, we analyzed four different filter algorithms as follows: (1) exact match, included inhibitory OTUs based on the full Sanger sequences (mean $1074.8 \mathrm{bp}$ ) of isolates that inhibited $B d$ growth (819 isolates clustering to 291 OTUs including 192 exclusively inhibitory OTUs); (2) trimmed exact match, included inhibitory OTUs based on sequences trimmed to the first 100-bp beyond primer 515f (5'-GTGCCAGCMGCCGCGGTAA-3') to match the Illumina reads (819 isolates clustering to 327 OTUs); (3) expected inhibitory OTUs expanded to include neighboring OTUs within 0.1 Jukes-Cantor distance on the Greengenes phylogenetic tree (7266 OTUs); (4) exclusively inhibitory OTUs (isolates within an OTU are all capable of significantly inhibiting $B d$ growth) expanded to include neighboring OTUs (6684 OTUs). This differs from the expected inhibitory OTUs and is more conservative because all tested isolates within an OTU must 


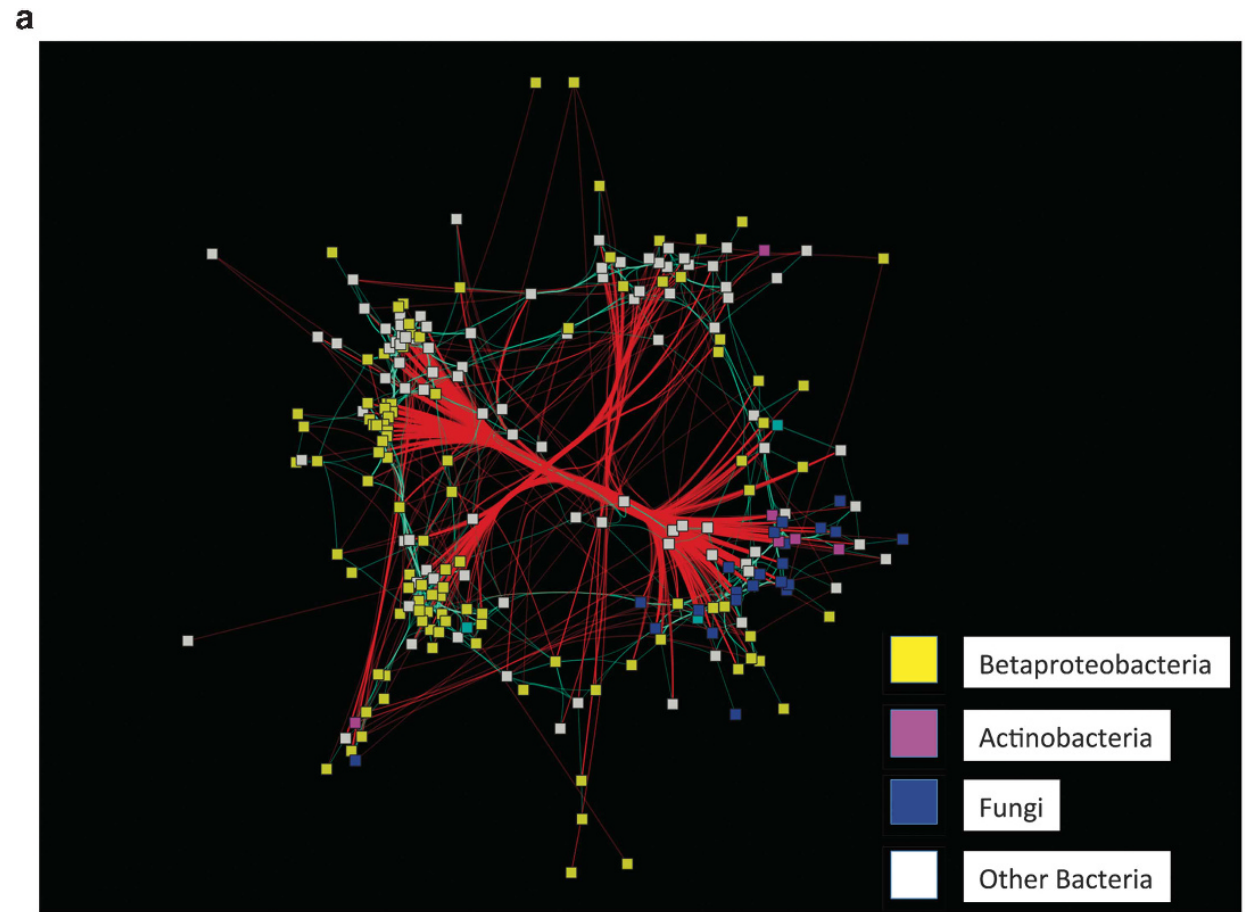

b

Fungal Class

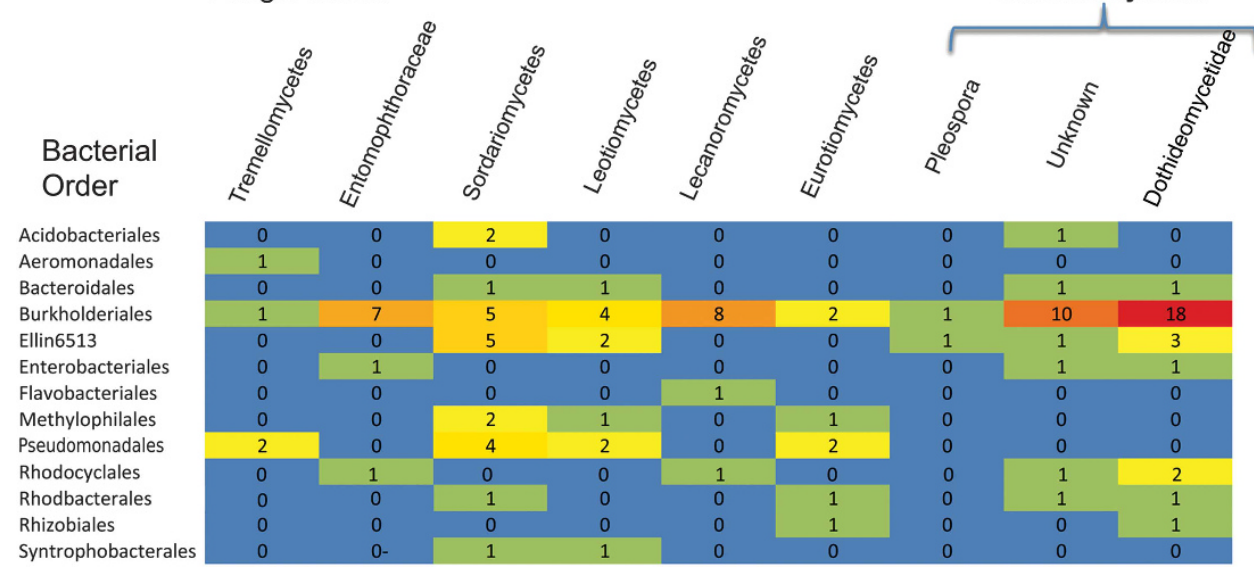

Figure 3 Analysis of co-occurring bacterial and fungal OTUs found on boreal toads at one site. This analysis included 19 toad individuals from Trout Creek for which we had successful sequencing yields for both $16 \mathrm{~S}$ and 18S: $n=5$ tadpoles, $n=4$ metamorphs, $n=5$ subadults and $n=5$ adults. (a) Network analysis depicting only significantly co-occurring bacterial and fungal OTUs. All square nodes represent OTUs (either bacteria or fungi) with significant co-occurrence with another OTU in the network. Red lines indicate negative co-occurrences between two OTUs. Turquoise lines indicate positive co-occurrences between two OTUs. Yellow = Betaproteobacteria, purple $=$ Actinobacteria, blue $=$ Fungi, white $=$ other bacterial OTUs. Bacteria are represented by taxa that were observed to be more abundant on tadpoles (Betaproteobacteria) or on adult stages (Actinobacteria, see Figure 1a). (b) Heatmap depicting the number of negatively co-occurring OTUs between bacterial and fungal taxa. (All positively correlated interactions are excluded from the heatmap, but are available in Supplementary Data via DRYAD). The numbers indicate the frequency of negative co-occurrences between bacterial and fungal groups calculated as the sum of negative OTU co-occurrences per bacterial order and fungal class, which are also depicted as red lines in a.

demonstrate antifungal function, rather than just some of the tested isolates within the OTU.

The use of expected inhibitory and exclusively inhibitory allows us to include closely related taxa that we hypothesize may also share antibiosis mechanisms with taxa known to inhibit fungi in culture. When inhibitory OTUs are mapped on the tree they cluster, suggesting they share these abilities; however, we do not have all possible OTUs from amphibians tested in culture. This approach can augment the culture database, for example, by inclusion of an OTU that falls closely between two OTUs with known antifungal ability.

We then filtered our bacterial OTU table in this boreal toad study to retain only the $B d$-inhibitory OTUs found on these lists. We compared the relative abundance of $B d$-inhibitory reads (out of 9625701 total Illumina sequencing reads) among the boreal 
toad samples including 80 adults, 32 juveniles, 47 metamorphs, 22 tadpoles and 15 lake-water samples from 7 sites, and 4 lake-sediment samples from 2 sites. These estimates of relative abundance of $B d$ inhibitory bacteria were compared among categories by Kruskal-Wallis tests.

\section{Results}

Sequencing results yielded 1972 unique bacterial OTUs and 500 unique microeukayotic OTUs. Boreal toad bacterial skin communities were predominately comprised of phyla Proteobacteria, Actinobacteria, Bacteroidetes, Acidobacteria and Verrucomicrobia. Bacteria found on $>90 \%$ of all subadult and adult samples consisted of many genera and included Pseudomonas, Janthinobacterium, Sphingomonos and Cellulosimicrobium. Cellulosimicrobium had the highest proportional abundance on adult toads. OTUs belonging to families Comamonadaceae, Pseudomonadaceae, Sphingomonadaceae, Promicromonosporaceae and Syntrophobacteraceae were found on all premetamorphic toads. Boreal toad eukaryotic communities were predominately comprised of classes of Fungi, Alveolata and Stramenopiles. No microeukaryotic OTUs were found across all life stages, however certain fungal groups (Dothideomycetes, Sordariomycetes and Entomophthoraceae) were found on all subadult and adult toads.

\section{Variation in toad skin microbiota}

We find that life stage, site and $B d$ presence or absence were all factors that explained a significant amount of variation in skin bacterial communities. To evaluate the factors that best explain the variation of toad skin communities we performed a PERMANOVA with life stage and $B d$ presence or absence as fixed effects and site as a random effect nested within $B d$ presence or absence (summary statistics can be found in Supplementary Table S2). Bacterial taxa that differed significantly by life stages, and $B d$ presence or absence can be found in Supplementary Material (on DRYAD). Life stage ( $P=0.001$, Figure 4$)$ and Site, $(P=0.001)$ described the greatest amount of variation.

Developmental transitions in taxa and alpha diversity Differences in proportional abundance of bacteria by life stage were driven by the replacement of one dominant taxon by another. Through development, tadpole skin-communities dominated by Betaproteobacteria are replaced by Actinobacteria dominated communities in adults. The proportional abundance of Betaproteobacteria in tadpoles is 62.4 percent compared with 10.9 percent in adults. The proportional abundance of Actinobacteria in tadpoles is 5.2 percent compared with 57.3 percent in adults. Other phyla, including Bacteroidetes, Acidobacteria and Verrucomicrobia, remained relatively constant

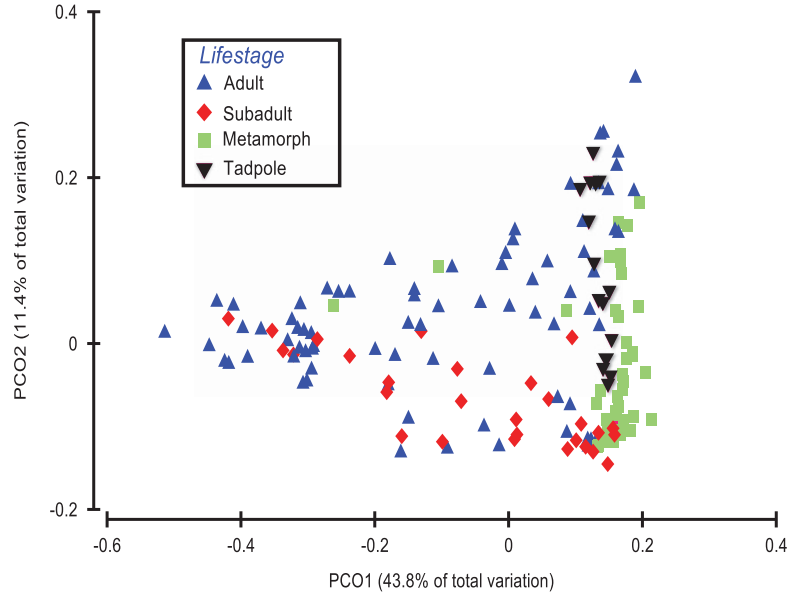

Figure 4 Beta diversity of skin bacterial communities of $A$. boreas from all sites, differentiated by life-stage. Dark blue $=$ adults $(n=77)$, red $=$ subadults $(n=28)$, green $=$ metamorphs $(n=47)$, black $=$ tadpoles $(n=16)$. Diversity patterns are visualized using principle coordinate plots of weighted UniFrac distances. Each point represents the skin bacterial community of an individual boreal toad; symbol color and shape indicate life-stage.

across developmental life stages (Figure 1a). Within our bacterial data set, there are overall significant differences in alpha diversity (Shannon index, richness and phylogenetic diversity) between tadpoles and all other life stages as well as between metamorphs and juveniles and adults, with Shannon index, $N=181$, ANOVA: $\left.\mathrm{F}_{3,177}=13.79, P<0.0001\right)$. Tadpoles held the lowest average diversity, followed by a spike in bacterial diversity on metamorphs that declined to adulthood. We found the same pattern within Trout Creek (Shannon diversity index, $P=0.0694$, Figure 2a). Quantitative PCR of bacterial DNA revealed tadpoles and metamorphs have reduced abundance of bacteria compared with juveniles and adults (Supplementary Figure S4).

For microeukaryotes sequenced from the boreal toads, the skin community of tadpoles initially dominated by the classes Alveolata and Stramenopiles becomes dominated by fungi. Fungi represent 3.3 percent proportional abundance in tadpoles compared with 52.1-57.0 percent of relative abundance in subdults and adults (Figure 1b). Higher taxonomic resolution of microeukaryotes shown across life stages can be seen in Supplementary Figure 5. We found 14 unique fungal OTUs on tadpoles, indicating that fungal taxa are present on tadpoles in low abundance and low richness. Alpha diversity of microeukaryotes was lowest in tadpoles and increases after metamorphosis. Significant differences among tadpoles, metamorphs, juveniles, and adults were observed (Shannon diversity index, ANOVA: $\mathrm{F}_{3,23.6}=15.17, P<0.0001$; Figure 2). No $B d$ was detected at this historically $B d$-free site, however the identical protocol has been used to detect $B d$ in other data sets. Quantitative PCR of fungal DNA revealed significant differences (Kruskal-Wallis test, $P=0.00526$ ) in abundance of fungi by life stage. 


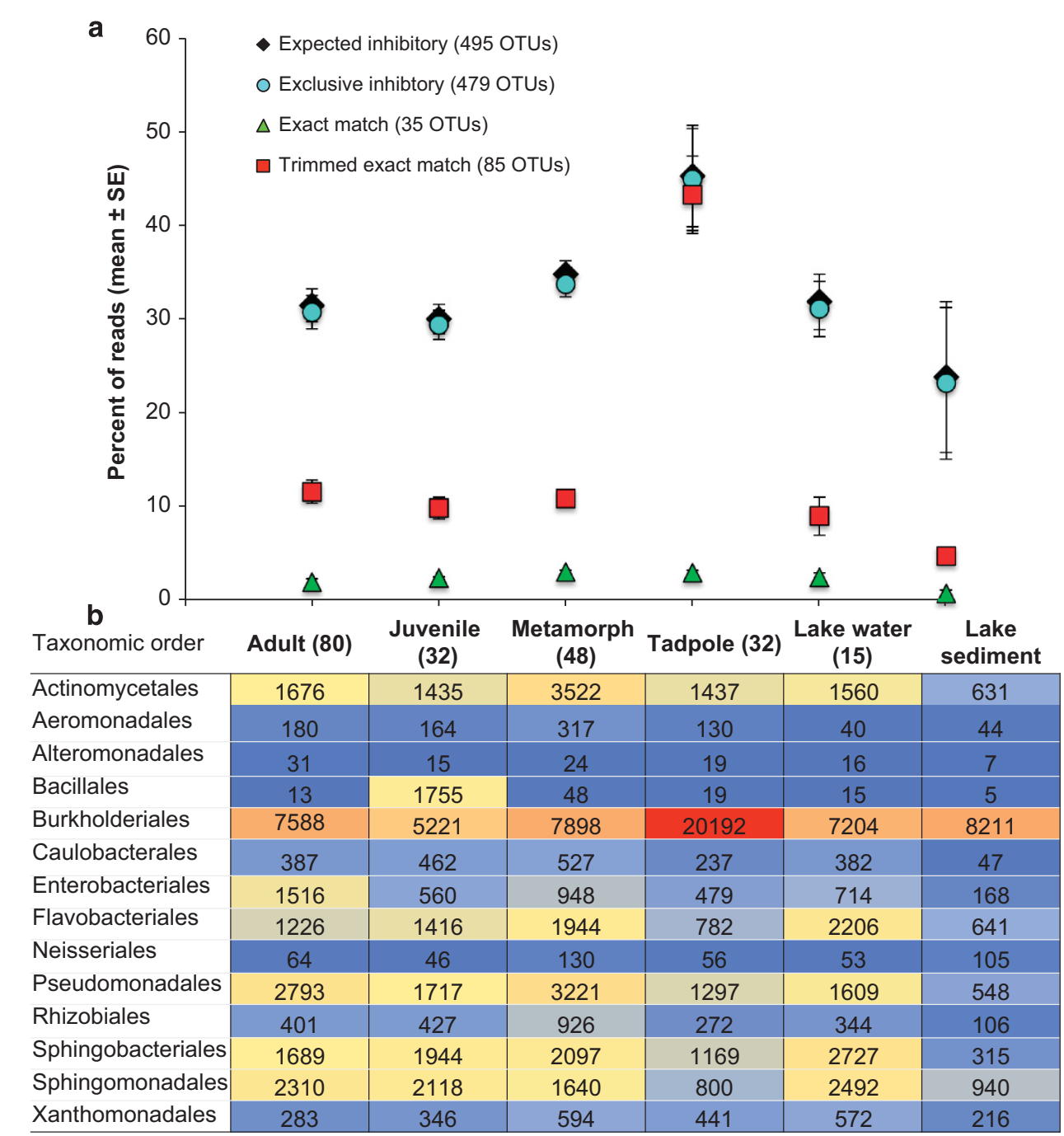

Figure $5 B d$-inhibitory OTUs found on boreal toads and in environmental samples. (a) Mean percent of bacterial sequencing reads with $B d$-inhibitory function, from boreal toad life-stages or environmental samples (across all sites, sample sizes in parentheses). $B d$-inhibitory function was determined by matching OTUs to the database described in the methods and Woodhams et al., 2015. Four different bioinformatic algorithms were used to identify $B d$-inhibitory OTUs as described in the methods section: exact match sequences, trimmed exact matches, exclusively inhibitory matches, and expected inhibitory matches. All four methods showed similar patterns and significant differences among sample type (Kruskal-Wallis tests, $P$ 's $<0.01$ ) with tadpoles, and to a lesser extent metamorphs, having a significantly higher percentage of their skin bacteria comprised of $B d$-inhibitory OTUs. (b) A heatmap showing the 14 bacterial taxa that contain the 'exclusively Bd-inhibitory matches' (479 OTUs, shown in aqua in Figure 5a) and how they are distributed across boreal toad lifestages and environmental samples. The lifestage and environmental sample $x$ axis labels apply to both a and $\mathbf{b}$.

Again, tadpoles and metamorphs have a reduced abundance of fungi compared with juveniles and adults.

\section{Co-occurrence networks}

The co-occurrence network of bacteria and eukaryotes found on Trout Creek toads revealed a large number of significant interactions. The co-occurrence network consists predominately of bacterial OTUs and bacteria-bacteria edges (16S-16S edges: $N=501$, 16S-18S: $N=177$, 18S-18S: $N=43$ ). Four major clusters are evident in the network (Figure 3a), with high intercluster coexclusion and intracluster соoccurrence. The network's large-scale patterns show fungal taxa mainly co-occurring with other fungal taxa (and some with Actinobacteria), and OTUs from the phylum Betaproteobacteria having significant interactions widely distributed throughout the entire network. Our data give greater awareness of interactions within communities, across life stages (for example, Proteobacteria interactions in tadpoles, as well as fungal-fungal interactions in post metamorphs (Figures 3a and b)).

\section{Detection of pathogen Bd (quantitative PCR)}

Sixteen individuals of $33(48.5 \%)$ from six $B d$-positive sites tested positive for the pathogen $B d$. These included 13 adults, 1 subadult and 2 
metamorphic toads. Individual infection intensities ranged from 2 DNA copies to 4840 DNA copies, with a mean $B d$ intensity of 583 DNA copies. Using Spearman correlation, we did not find any bacterial OTUs that correlated with infection intensity.

\section{Bd-inhibitory bacterial OTUs}

After OTU filtering using the $B d$-inhibitory bacterial OTUs database (Woodhams et al., 2015), we found 35 OTUs with exact matches and 85 OTUs with trimmed exact matches within the boreal toad data set, 495 expected inhibitory OTUs, and 479 exclusively inhibitory OTUs using the expanded phylogenetic estimation. We find a significant difference in the proportion of known inhibitory sequences by life stage, and the same pattern for all inhibitory data sets by Kruskal-Wallis test: exact match $P<0.001$, trimmed exact match $P<0.001$, expected inhibitory $P=0.005$, and exclusively inhibitory $P=0.006$, (Figure 5a). Tadpoles hosted significantly more inhibitory OTUs than other life stages or environmental samples (Figure 5a). The differential abundance of inhibitory taxa across life stages is most pronounced using the trimmed exact-match approach. When we refined this analysis to compare infected adult individuals $(N=16)$ with uninfected adults $(N=73)$ from 17 different sites, we observed a marginally significantly higher percentage of the microbiome included inhibitory OTUs on the uninfected adults, as determined by Mann-Whitney $U$ tests. This was the case when we used either the exclusive matches to the inhibitory database $(P=0.045$; uninfected adults had an average of $32.1 \%$ of the sequences matching $B d$-inhibitors, while infected adults had $23.6 \%$ ) or the expected matches $(P=0.051$; uninfected adults had an average of $32.9 \% \mathrm{Bd}$ inhibitors, whereas infected adults had $24.3 \%$ ). Within Colorado, Chaffee County is the only remaining region with abundant and historical numbers of boreal toads. When we analyze this unique region compared with all other Colorado samples, we find that Chaffee County toads have a significantly higher abundance of expected and exclusively $B d$-inhibitory bacterial OTUs; expected $t=-3.12, \quad \mathrm{df}=78, \quad P=0.003 ; \quad$ exclusive: $t=-3.04$, $\mathrm{df}=78, P=0.003$.

\section{Discussion}

Our knowledge of amphibian skin microbiomes is rapidly increasing (Jani and Briggs, 2014; Kueneman et al., 2014; Walke et al., 2014). Exploring skin bacterial communities across different amphibian developmental stages and the defensive functions those microbes play, particularly prior to the development of the adaptive immune function, is an important and understudied area. Molecular methods for investigating microeukaryotes are just emerging, allowing us to further consider the role of nonbacterial microbes on the skin. In addition, there are few next-generation microbial data sets that compare $B d$-positive and $B d$-negative sites (Jani and Briggs, 2014). Within this study we begin to fill these knowledge gaps including describing $A$. boreas skin communities across developmental stages from $B d$-positive and -negative sites across the host range in Colorado; surveying microeukaryotes from amphibians utilizing high throughput sequencing; and providing the first application of a cultureindependent database of known and expected $B d$-inhibitory isolates. Our data suggest that host development is the central driver of $A$. boreas skin communities, and that both positive and negative interactions amongst and between the bacterial and microeukaryotic taxa are occurring on the amphibian skin. In particular, tadpole skin communities appear to be restrictive in their community types, harboring an abundance of known $B d$-inhibitory bacteria.

\section{Factors explaining variation in the boreal toad skin microbiome}

Developmental life stage, site (wetland site of collection), and $B d$ presence or absence at a site were all significant predictors of skin microbial communities. However, developmental life stage has an effect size that is twice as large as site and three and a half times as large as $B d$ presence, suggesting that life stage was the most important factor structuring microbial communities on boreal toads (Supplementary Table S2). Discussion of OTUs that significantly differentiate infected vs uninfected sites is found in Supplementary Material. Intriguingly, microbial communities belonging to the one remaining Colorado County with persisting Boreal toad populations was shown to have a unique microbial signature (Supplementary Material).

\section{Skin bacterial and microeukaryote diversity across} boreal toad developmental stages

The skin-associated microbial communities vary significantly across boreal toad developmental stages (Figures 1a and b, 4). First, we will summarize the patterns in skin bacteria, by life stage and below we will discuss the microeukaryotes. Bacteria on tadpoles were dominated by Proteobacteria. The taxonomic group Proteobacteria is almost entirely comprised of one OTU from the family Comomonadaceae, which is not further identified and represents a very diverse group of bacteria with known $B d$-inhibitory members. Curvibacter, a member of Comamonadaceae, was also found to be the most dominant member of larval stages in Tiger salamanders (Ambystoma tigrinum), Western chorus frogs (Pseudacris triseriata), and Northern leopard frogs (Lithobates pipiens) in Colorado (McKenzie et al., 2011). Compared to other life stages, tadpoles of A. boreas had the lowest alpha diversity scores for both bacteria and microeukaryotes (Figures 2a and b). The pattern of alpha diversity by life stage 
suggests that microbial colonization at the tadpole stage is restricted and that the community undergoes a restructuring after metamorphosis. Evidence supporting reduced and bacterially restrictive communities was also found for tadpoles of frog species Rana cascadae (Kueneman et al., 2014). Tadpoles of $R$. cascadae had microbial communities with lower alpha diversity than the lake water they inhabited, suggesting that tadpoles of some species maintain restrictive bacterial communities compared to other developmental stages.

We observed the highest levels of bacterial species richness and evenness on samples collected just after metamorphosis (Figure 2a). Intriguingly, quantitative PCR of bacteria shows that bacterial DNA abundance is lowest in metamorphs, revealing that high diversity is coupled with low abundance of bacteria in metamorphs. Bacterial abundance is also low in tadpoles and is significantly higher in subadults and adults (Supplementary Figure S4). Undoubtedly during metamorphosis, organ transformation and the restructuring of the immune system is an energetically costly process; one hypothesis is that regulation of skin microbial communities during the disturbance of metamorphosis is energetically challenging. These factors, coupled with skin shedding during metamorphosis, may allow for an open niche, explaining the observed jump in alpha diversity in metamorphs.

Skin bacterial communities of $A$. boreas subadults and adults do not significantly differ from one another and are almost entirely comprised of unidentified species of the phylum Actinobacteria, genus Cellulousmicrobium, which are Gram-positive non-motile, rod-shaped or coccoid, bacterial strains. Described species have been isolated from soil (Yoon et al., 2007).

Microeukaryotes are substantially more diverse in subadults and adults compared with tadpoles and metamorphs. The increase in the diversity of microeukaryotes is marked by a transition to a fungi-dominated community (Figures $1 \mathrm{~b}$ and $2 \mathrm{~b}$ ). It appears that fungi preferentially colonize the skin of post-metamorphic amphibians, suggesting that bacterial communities of tadpoles are able to restrict colonization of fungi. Some fungi of amphibians are known to infect keratinized skin of postmetamorphic amphibians, however, this work demonstrates that other fungi can establish in the skin community of larval tadpoles which typically do not have keratinized skin. Quantitative measurement of fungal DNA across taxa reveal that fungal DNA is low but present in tadpoles and metamorphs and significantly increased in subadults and adults (Supplementary Figure S4). It remains unknown whether native fungal diversity may help to exclude invasive fungal pathogens and help explain why adults often survive epidemics, which disproportionately kill metamorphs and juveniles. In our analysis of co-occurrence, we did not find any fungi that negatively co-occur with one another.
Bacterial and fungal interaction in the boreal toad skin microbiome

Using co-occurrence network analysis on four life stages from Trout Creek, we detect many significant negative and positive correlations between fungal OTUs and bacteria and fungal OTUs (Figure 3a). However, as co-occurrence data are correlational in nature, coculturing or in vitro studies are necessary to confirm significant interactions. Co-occurrence analyses can suggest important relationships between taxa that might be owing to a variety of ecological interactions between taxa. From this network analysis, we observe that 58 out of 116 $(50 \%)$ of the negative co-occurrence interactions between bacteria and fungi occurred with bacterial OTUs belonging to the Burkholderiales (primarily in the family Comamonadaceae). Furthermore, the Burkholderiales OTUs negatively co-occurred with all nine of the major fungal taxa examined here (Figure 3b). The Pseudomonadales OTUs with significant negative co-occurrences are also noticeable with several OTUs negatively co-occurring with nearly half of the fungal groups examined here. These findings from the network analysis agree with previous culturing work using challenge assays against fungi (synthesized in Woodhams et al., 2015), such that those studies also find isolates belonging to Burkholderiales and Pseudomonadales highly represented amongst those that prove to be fungal inhibitors (for example, Janthinobacterium lividum and Pseudomonas veronii). For several other bacterial groups analyzed here, we know less about their ability to inhibit fungi from culture-based studies. Bacterial OTUs and their interactions with fungal OTUs are deposited in DRYAD. Positive associations between fungi and bacteria were most abundant in Actinomycetales and Sphingomonadales, bacteria that were most abundant on adult toads. Bacterial OTUs that associate positively with fungal groups are deposited in DRYAD.

\section{Distribution of Bd-inhibitory OTUs across boreal toad life stages and environment}

We identified bacteria in our data set with expected $B d$-inhibitory function using a bioinformatics approach, including matching boreal toad sequences to a database of cultured isolates tested in vitro for ability to inhibit the growth of $B d$ (Woodhams et al., 2015). We found that using direct sequence matches between cultures and next-generation sequencing or by matching next-generation sequences with an expanded list of $B d$-inhibitory OTUs based on phylogenetic proximity provided similar results. Significant differences in $B d$-inhibitory bacteria occurred among life stages of boreal toad and environmental samples. Specifically, tadpoles are enriched with $B d$-inhibitory bacteria (Figure 5a) and this is corroborated by a lack of fungi in $18 \mathrm{~S}$ next-generation sequences from tadpoles (Figure $1 \mathrm{~b}$ and Supplementary Figure S4). Taxonomic 
groups including Burkholderiales, Actinomycetales, Pseudomonadales and Sphingomonadales were prevalent $B d$-inhibitory bacteria (Figure $5 b$ ) that were also negatively co-occurring with fungi in our network analysis (Figure 3b). This information suggests that probiotic approaches to disease management (Harris et al., 2009; Bletz et al., 2013) have merit and that enrichment for $B d$-inhibitory bacteria may be a host strategy to boost defenses against fungal pathogens, particularly during early developmental stages. Surprisingly we did not find $B d$-inhibitory bacteria to significantly differentiate by $B d$ presence or absence at a site or by infection intensity. Selection for $B d$-inhibitory bacteria may have been masked by overall skin variation in adults and juveniles. Beta diversity measure showed a large degree of within life stage variation for adults and juveniles (Figure 4, Supplementary Figure S3). Small sample size of infected toads $(N=16)$ may have limited our ability to detect differences in bacterial OTUs that may correlate with infection. Last, other intrinsic factors, such as site effects, may have swamped out the finer details of $B d$ inhibition. We do, however, find higher levels of $B d$-inhibitory bacteria in Chaffee County. This county still maintains several robust populations and thus is unique in Colorado.

The results of our study demonstrate that the skinassociated microbial communities of boreal toads are strongly dictated by developmental stage of the host, with site variation and $B d$ pathogen presence also as contributing factors. By examining both the bacterial and microeukaryotic members of the skin community, we have results that suggest that larval, early life stages of boreal toads rely heavily on the skin microbiome for reducing colonization of pathogens and 'undesired' microbes until the development of a more fully functioning adaptive immune system following metamorphosis. Thus the skin microbiome acts as an externally functioning immune partner during the early life stages of $A$. boreas. Several results from this study support this conclusion. First, analysis of similarity reveals tadpole skin communities have the least amount of within group variation and are distinct from other life stages (Figure 4, Supplementary Figure S2). Second, Shannon diversity of both bacteria and fungi were lowest in tadpoles (Figures 2a and b). Third, tadpole's communities are lacking in fungal taxa best explained by bacterial-fungal interactions (Figures 1b and 2b). Fourth, bacterial OTUs that matched known $B d$-inhibitory isolates were proportionally more abundant on tadpoles (Figure 5b).

Novel strategies may be required to ensure the persistence of endangered Colorado A. boreas. Manipulations of skin microbial communities have been proposed as one possible tool, coupled with reintroductions to improve survival (Bletz et al., 2013). We highlight taxa that associate with disease at sites, however experimental work is needed to confirm associations between host-associated microbial communities and disease. Understanding the relationships that have evolved between amphibians and their skin microbiota offers an opportunity to develop therapeutic tools for one of the world's most threatened taxa.

\section{Conflict of Interest}

The authors declare no conflict of interest.

\section{Acknowledgements}

We acknowledge Colorado Parks and Wildlife for assistance in sample collection and $B d$ prevalence data at sample sites. In particular, Kevin Thompson, Boyd Wright, Jennifer Logan and Paul Jones assisted in sample collection and site access. Alba Diana Vilar provided extraordinary field help. Thanks to Antonio Gonzalez, Sophie Weiss, and Tony Walters for computational assistance, and Laura Wagner Parfrey, for help with filtering 18s data. This project was supported by an NSF grant (DEB: 1146284) to VJM and RK and in part by a grant from the John S. Templeton Foundation to VJM and RK. JGK was supported by an NSF graduate fellowship and received funds from the University of Colorado EBIO departmental summer grants.

\section{References}

Banning JL, Weddle AL, Wahl GW 3rd, Simon MA, Lauer A, Walters RL et al. (2008). Antifungal skin bacteria, embryonic survival, and communal nesting in four-toed salamanders, Hemidactylium scutatum. Oecologia 156: 423-429.

Benjamini Y, Hochberg Y. (1995). Controlling the false discovery rate: a practical and powerful approach to multiple testing. JR Stat Soc Ser B 57: 289-300.

Berry D, Widder S. (2014). Deciphering microbial interactions and detecting keystone species with co-occurrence networks. Front Microbiol 5: 219.

Bletz MC, Loudon AH, Becker M, Bell SC, Woodhams DC, Minbiole KPC et al. (2013). Mitigating amphibian chytridiomycosis with bioaugmentation: characteristics of effective probiotics and strategies for their selection and use. Ecol Lett 16: 807-820.

Brucker RM, Harris RN, Schwantes CR, Gallaher TN, Flaherty DC, Lam BA et al. (2008). Amphibian chemical defense: antifungal metabolites of the microsymbiont Janthinobacterium lividum on the salamander Plethodon cinereus. J Chem Ecol 34: 1422-1429.

Emerson JB, Keady PB, Brewer TE, Clements N, Morgan EE, Awerbuch J et al. (2015). Impacts of flood damage on airborne bacteria and fungi in homes after the 2013 Colorado Front Range flood. Environ Sci Technol 49: 2675-2684.

Faust K, Raes J. (2012). Microbial interactions: from networks to models. Nat Rev Microbiol 10: 538-550.

Friedman J, Alm EJ. (2012). Inferring correlation networks from genomic survey data. PLoS Comput Biol 8: e1002687.

Goettl JP Jr (1997). Boreal Toad (Bufo boreas) Recovery Plan (Southern Rocky Mountain Population). Division of Wildlife: Denver, CO, USA, p 45. 
Harris RN, Brucker RM, Walke JB, Becker MH, Schwantes CR, Flaherty DC et al. (2009). Skin microbes on frogs prevent morbidity and mortality caused by a lethal skin fungus. ISME J 3: 818-824.

Hooper LV, Littman DR, Macpherson AJ. (2012). Interactions between the microbiota and the immune system. Science 336: 1268-1273.

Iwasaki A, Ruslan M. (2010). Regulation of adaptive immunity by the innate immune system. Science 327: 291-295.

Jani AJ, Briggs CJ. (2014). The pathogen Batrachochytrium dendrobatidis disturbs the frog skin microbiome during a natural epidemic and experimental infection. Proc Natl Acad Sci USA 111: E5049-E5058.

Kilpatrick AM, Briggs CJ, Daszak P. (2010). The ecology and impact of chytridiomycosis: an emerging disease of amphibians. Trends Ecol Evol 25: 109-118.

Kueneman JG, Parfrey LW, Woodhams DC, Archer HM, Knight R, McKenzie VJ. (2014). The amphibian skinassociated microbiome across species, space and life history stages. Mol Ecol 23: 1238-1250.

McKenzie VJ, Bowers RM, Fierer N, Knight R, Lauber CL. (2011). Co-habiting amphibian species harbor unique skin bacterial communities in wild populations. ISME J 6: $588-596$.

Murphy PJ, St-Hilaire S, Bruer S, Corn PS, Peterson CR. (2009). Distribution and pathogenicity of Batrachochytrium dendrobatidis in boreal toads from the Grand Teton area of western Wyoming. EcoHealth 6: 109-120.

Muths E, Guyer C. (2003). Home range and movements of boreal toads in undisturbed habitat. Copeia 1: 160-165.

Olson DH. (1992). Ecological susceptibility of amphibians to population declines. In Proceedings of Symposium on Biodiversity of Northwestern California. Davis, CA: University of California Wildland Resources Center Report 29: 55-62.

Rautava S, Luoto R, Salminen S, Isolauri E. (2012). Microbial contact during pregnancy, intestinal colonization and human disease. Nat Rev Gastroenterol Hepatol 9: 565-576.

Rollins-Smith LA, Ramsey JP, Pask JD, Reinert LK, Woodhams DC. (2011). Amphibian immune defenses against chytridiomycosis: impacts of changing environments. Integr Comp Biol 51: 552-562.

Rollins-Smith LA, Ramsey JP, Reinert LK, Woodhams DC, Livo LJ, Carey C. (2009). Immune defenses of Xenopus laevis against Batrachochytrium dendrobatidis. Front Biosci 1: 68-91.

Rollins-Smith LA. (1998). Metamorphosis and the amphibian immune system. Immunol Rev 166: 221-230.

Savage AE, Zamudio KR. (2011). MHC genotypes associate with resistance to a frog-killing fungus. Proc Natl Acad Sci USA 108: 16705-16710.

Scherer RD, Muths E, Lambert BA. (2008). Effects of weather on survival in populations of boreal toads in Colorado. J Herpetol 42: 508-517.

Scherer RD, Muths E, Noon BR, Corn PS. (2005). An evaluation of weather and disease as causes of decline in two populations of boreal toads. Ecol Appl 15: 2150-2160.

Scherff-Norris KL. (1997). Hatchery Manual for the Rearing and Propagation of Captive Boreal Toads, Bufo Boreas. Division of Wildlife: Denver, CO, USA, p 21.

Smoot ME, Ono K, Ruscheinski J, Wang PL, Ideker T. (2011). Cytoscape 2.8: new features for data integration and network visualization. Bioinformatics 27: 431-432.

Tennessen JA, Woodhams DC, Chaurand P, Reinert LK, Billheimer D, Shyr Y et al. (2009). Variations in the expressed antimicrobial peptide repertoire of northern leopard frog Rana pipiens populations suggest intraspecies differences in resistance to pathogens. Dev Comp Immunol 33: 1247-1257.

Walke JB, Becker MH, Loftus SC, House LL, Cormier G, Jensen RV et al. (2014). Amphibian skin may select for rare environmental microbes. ISME J 8: 2207-2217.

Walke JB, Harris RN, Reinert LK, Rollins-Smith LA, Woodhams DC. (2011). Social immunity in amphibians: evidence for vertical transmission of innate defenses. Biotropica 43: 396-400.

Woodhams DC, Alford RA, Antwis RE, Archer H, Becker $\mathrm{MH}$, Belden LK et al. (2015). Antifungal isolates database of amphibian skin-associated bacteria and function against emerging fungal pathogens. Ecol Arch 96: 595.

Woodhams DC, Brandt H, Baumgartner S, Kielgast J, Küpfer E, Tobler U et al. (2014). Interacting symbionts and immunity in the amphibian skin mucosome predict disease risk and probiotic effectiveness. PLOS One 9: e96375.

Yoon JH, Kang SJ, Schumann P, Oh TK. (2007). Cellulosimicrobium terreum sp. nov., isolated from soil. Int $J$ Syst Evol Microbiol 57: 2493-2497.

Supplementary Information accompanies this paper on The ISME Journal website (http://www.nature.com/ismej) 